\section{Trends in epidemiology in the 21st century: time to adopt Bayesian methods}

\author{
Tendências da epidemiologia no século XXI: \\ é o tempo dos métodos bayesianos
}

\author{
Tendencias de la epidemiología del siglo XXI: \\ es tiempo para los métodos bayesianos
}

\begin{abstract}
2013 marked the 250th anniversary of the presentation of Bayes' theorem by the philosopher Richard Price. Thomas Bayes was a figure little known in his own time, but in the 20th century the theorem that bears his name became widely used in many fields of research. The Bayes theorem is the basis of the so-called Bayesian methods, an approach to statistical inference that allows studies to incorporate prior knowledge about relevant data characteristics into statis tical analysis. Nowadays, Bayesian methods are widely used in many different areas such as astronomy, economics, marketing, genetics, bioinformatics and social sciences. This study observed that a number of authors discussed recent advances in techniques and the advantages of Bayesian methods for the analysis of epidemiological data. This article presents an overview of Bayesian methods, their application to epidemiological research and the main areas of epidemiology which should benefit from the use of Bayesian methods in coming years.
\end{abstract}

Bayes Theorem; Statistics; Probability Theory
Edson Zangiacomi Martinez 1 Jorge Alberto Achcar 1

\section{Resumo}

O ano de 2013 marca o 250o aniversário da apresentação do teorema de Bayes pelo filósofo Richard Price à Royal Society em 1763. Thomas Bayes foi uma pessoa pouco conhecida em sua época, mas no século XX o teorema que leva o seu nome tornou-se amplamente utilizado em muitas áreas de pesquisa. O teorema de Bayes é a base dos chamados métodos bayesianos, um procedimento de inferência estatística que permite incorporar na análise o conhecimento prévio sobre características relevantes dos dados. Atualmente, os métodos bayesianos são largamente usados em muitas diferentes áreas como astronomia, economia, marketing, genética, bioinformática e ciências sociais. Em adição, é observado na literatura que muitos autores têm discutido os recentes avanços do uso dos métodos bayesianos na análise de dados epidemiológicos. No presente artigo, apresentamos uma visão global dos métodos bayesianos, sua utilidade na pesquisa epidemiológica e os tópicos em epidemiologia em que estes métodos podem ser extensivamente usados nos próximos anos.

Teorema de Bayes; Estatística; Teoria da Probabilidade 


\section{Introduction}

The first mathematical formulation using the Bayesian method is attributed to Thomas Bayes, a British Presbyterian minister. Very little is known about his personal history. It is believed that he was born around 1701 in Hertfordshire, England and died in 1761 in Tunbridge. Many facts about his life are speculation such as the exact date of his birth and the authorship of a book on Theology entitled Divine Benevolence: or an Attempt to Prove That the Principal End of the Divine Providence and Government is the Happiness of His Creatures, that concerned the motive behind God's actions in making the world. In 1719, he began his studies of logic and theology at the University of Edinburgh. The only scientific work published during his lifetime was The Doctrine of Fluxions, in 1736, in which he defended the logical foundation of Isaac Newton's calculus. Two years after his death, his friend Richard Price (1723-1791) presented the Royal Society with a manuscript authored by Thomas Bayes entitled An Essay Towards Solving a Problem in the Doctrine of Chances ${ }^{1}$. Price said he found the essay among Bayes' papers and in his opinion it "has great merit and well deserves to be preserved" 2 (p. 451). The essay offered the first clear solution to a problem of inverse probability, where Bayes described how we can calculate the probability of the occurrence of an event given the known probability of a certain condition. This formula is known as Bayes' theorem. It is interesting to note that Richard Price believed that Bayes' theorem was based on theological arguments and it could prove the existence of God 3 . In 1748, the Scottish empiricist philosopher David Hume published a book entitled An Enquiry Concerning $\mathrm{Hu}$ man Understanding. In chapter ten of this work entitled of Miracles, Hume wrote his famous argument against miracles ${ }^{4}$. Today, some authors claim that Hume's statements were based on arguments taken from Bayes's theorem 5,6,7. Despite these philosophical ideas, Bayes' essay seemed to have been forgotten until the publication of the book entitled Théorie Analytique des Probabilités by the French mathematician and astronomer Pierre-Simon Laplace, in 1812. It is believed that Laplace was not familiar with the work of Thomas Bayes and he independently developed a more formal version of Bayes' theorem.

Currently, Bayesian ideas are used in many fields of technology and research, such as modern computers which use Bayesian filters to classify emails and detect spam ${ }^{8}$. Another example of the modern use of Bayesian ideas is in robots which, based on a Bayesian framework 9 and a Bayes network based system, distinguished ter- restrial rocks from meteorites in the first robotic identification of a meteorite in 2000 in the Elephant Moraine in the Antarctic 10. In addition, NASA's Mars Exploration Rover mission has been using Bayesian classification algorithms to study the physical properties of the surface of Mars 11 . Nowadays, Bayesian methods are widely used in many different fields of research, such as astronomy 12, economics and econometrics 13,14, marketing 15 , actuarial science 16 , psychological research 17, genetics 18,19 , evolutionary biology 20 , bioinformatics 21 , demography 22 , social sciences 23 , public health 24 , drug development 25 and clinical trials 26,27 . The use of Bayesian methods in epidemiological studies has been discussed by several authors 28,29,30,31 and Congdon 32 claims that the Bayesian approach is very useful for modeling epidemiological datasets, since they allow the control of possible confounding influences on disease outcomes and the establishment of causal and dose-response relationships. In addition, Dunson 28 showed that the use of Bayesian techniques in epidemiological studies is a powerful mechanism for incorporating information from previous studies and controlling confounding. Appropriate methods for dealing with interactions between variables and confounding effects are essential for epidemiological studies, and in this respect Bayesian methods can be very useful. Bayesian methods represent a totally different way of thinking about research methods where the researcher's previous knowledge and experience have an important effect on inference and decision-making.

The traditional approach to statistical inference is the frequentist (or classical) technique, where results are interpreted in terms of the frequency of occurrence of an event observed in a hypothetically large number of repetitions of the experiment. Frequentist inferences are based only on observational data, while Bayesian inference assumes that prior knowledge can be formally incorporated into the analytical process. We can therefore say that the Bayesian research method is based both on an empirical world represented by the sample data and on human reasoning represented by the accumulated experience of the researcher.

In the present article, we present an overview of the Bayesian approach together with a brief description of the Bayesian statistical inference procedure and comparison with the standard frequentist approach. We also discuss the advantages of the Bayesian approach over the traditional research method applied to the analysis of epidemiological data and discuss some areas of epidemiology which should benefit from the use of Bayesian methods in coming years. 


\section{Are we in the Bayesian era?}

In 1996, David Moore published an article 33 that discussed the possibility of teaching Bayesian inference on a first statistics course for students from different backgrounds. He argued that Bayesian methods were rarely used in practice and teaching them would deprive students of instruction about more common statistical methods. It is possible that this statement was based on limitations caused by the time needed for software and hardware to analyze data using a Bayesian approach. Major advances in software and hardware in the last 20 years have been one of the factors that has led to a sharp increase in the use of Bayesian methods. To obtain an idea of the current use of Bayesian methods in health research, a search was made in PubMed using the keyword "Bayesian". The annual number of articles is presented graphically in Figure 1 which shows that the first article using the term "Bayesian" indexed in PubMed was published in 1963 34. After this first publication, we observe a very modest increase in the number of articles up to the middle of the 1980s, after which a large increase can be observed. It is important to remember that portable personal computers only became popular in the middle of the 1980s, significantly contributing to the use of Bayesian methods, since the approach is usually depends on computational algorithms. A large increase in the number of published articles using the term Bayesian can be observed toward the end of $20^{\text {th }}$ century. It is possible that this increase was due to the emergence of new software adapted to Bayesian analysis, such as the free software WinBUGS 35. This software uses simulation methods, such as the popular Markov Chain Monte Carlo (MCMC) methods 36 , and was possibly the most important computational advances to have popularized the use of Bayesian methodology. The first version of WinBUGS for Windows was made available in 1997 37. Today, OpenBUGS is the open-source version of WinBUGS and can be freely downloaded from the project website (http://www.openbugs.info/w/Downloads).

In 2010, 2.56 in every 1,000 articles indexed in PubMed contained the term Bayesian (Figure 1), showing the growing use of Bayesian methods in health research since the publication of Moore's article 29 and suggesting that Bayesian statistics is actually an important issue to students who are starting their studies to become researchs. The advantages of the use of Bayesian methods in specific fields of knowledge, such as genetics 18 , oncology 38 and para-

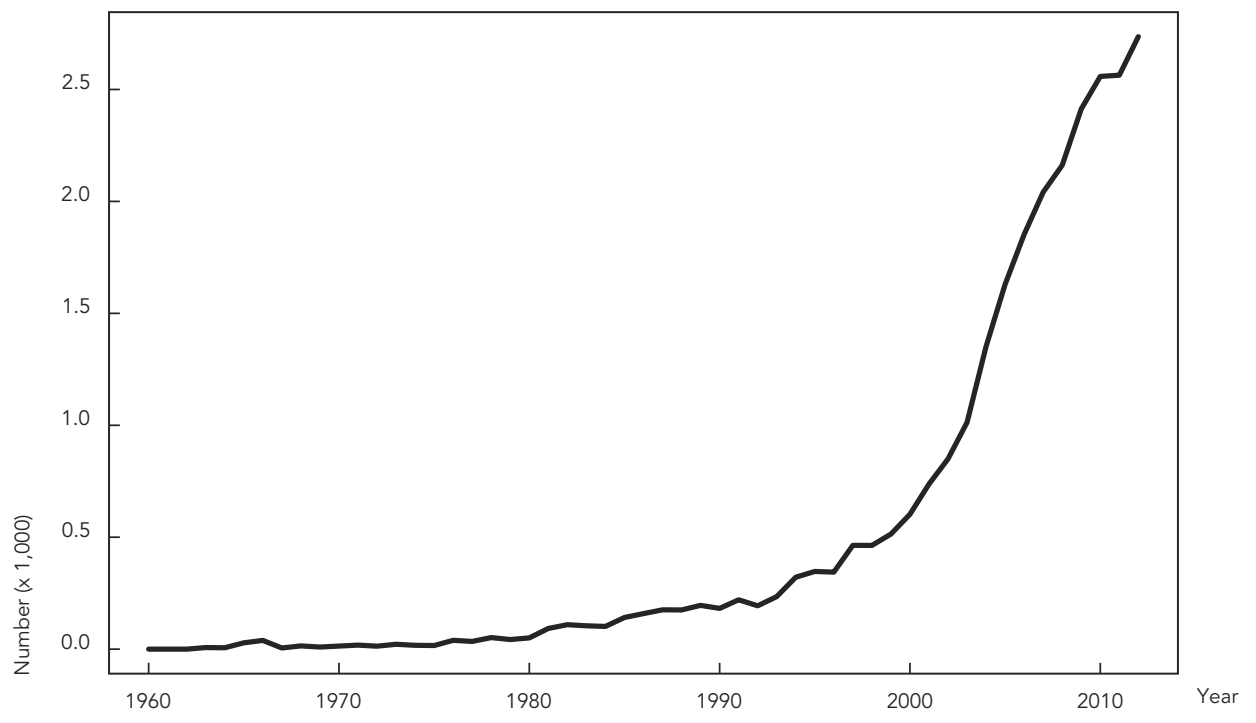


sitology 39, have therefore been discussed in a number of research articles available in the literature.

One of the reasons for the widespread use of Bayesian methods may be related to the ease with which statistical inferences can be made even with complex problems. It is therefore expected that the use of Bayesian methods will continue to increase in response to the demands of ever more complex problems in the health field.

\section{A practical example: estimating disease prevalence}

In order to illustrate Bayesian inference procedures, let us consider a simple example in which we estimate the prevalence $\theta$ of a disease among the inhabitants of a given community. A parameter is defined as an unknown numerical characteristic of a population. Prevalence $\theta$ is therefore a parameter and may be estimated using a frequentist or Bayesian approach. First, let us describe how this is done using the frequentist approach. A sample of size $n$ is represented by a probability function, defined as the likelihood function and denoted by $f(\boldsymbol{x} \mid \theta)$. In the frequentist approach, inference is based only on the likelihood function. $X_{i}$ is a random binary variable which assumes the value of 1 if the $i$ th individual has the disease of interest and the value of 0 if the $i$-th individual does not have the disease: $(i=1, \ldots, n)$. Thus, the probability of the $i$-th individual having the disease is $P\left(X_{i}=1\right)=$ $\theta$, and the probability of the individual not having the disease is $P\left(X_{i}=0\right)=1-\theta$, where $0 \leq \theta \leq 1$. In this case, we say that $X_{i}$ follows a Bernoulli distribution with success probability $\theta$, and its probability function is given by $P\left(X_{i}=x_{i}\right)=\theta x i$ $(1-\theta)^{1-x i}$, where $x_{i}$ assumes the value 0 or 1 . Assuming that $X_{1}, X_{2}, \ldots, X_{n}$ are independent random variables, that is, assuming that an individual having the disease does not affect the probability of another individual having the disease, the likelihood function is given by

$$
f(x \mid \theta)=\prod_{i=1}^{n} P\left(X_{i}=x_{i}\right)=\prod_{i=1}^{n} \theta^{x_{i}}(1-\theta)^{1-x_{i}}=\theta^{\sum_{i=1}^{n} x_{i}}(1-\theta)^{n-\sum_{i=1}^{n} x_{i}} .
$$

The maximum-likelihood estimation (MLE) procedure is a frequentist method commonly used to estimate the parameters of a statistical model 40,41. Using the MLE method the estimate of a parameter $\theta$ is given by the value of this parameter in the parameter space (set of all possible values of the parameter) that maximizes the likelihood function $f(x \mid \theta)$. For this purpose, we can use differential calculus tools to obtain an estimator $\hat{\theta}$ of $\theta$. In practice, it is often more convenient to maximize the logarithm of the likelihood function, also called the log-likelihood function. If we set the first derivative of the log-likelihood function equal to zero, the maximum likelihood estimator $\hat{\theta}$ of $\theta$ is given by

$\hat{\theta}=\frac{\sum_{i=1}^{n} x_{i}}{n}=\frac{\text { number of individual with the disease in the sample }}{\text { total number of individuals in the sample }}$

This is a well-known expression which can be found in many widely used epidemiology textbooks. Inference is then based on a hypothetical series of data sets collected under identical conditions. For example, a 95\% confidence interval is a range of values calculated from the sample observations, with $95 \%$ certainty that all possible random samples drawn from the same population using the same sampling scheme would generate intervals containing the true value of the parameter. However, a 95\% confidence interval does not mean that there is a $95 \%$ probability that the calculated interval contains the true value of the parameter. Although this interpretation is quite intuitive, it is not valid since the frequentist method cannot assign probabilities to any particular parameter.

The frequentist approach assumes that the parameter of interest is a fixed quantity, while in the Bayesian approach parameter uncertainty is represented by a probability distribution. From a statistical viewpoint, this is perhaps the most striking difference between the traditional frequentist approach and the Bayesian approach and is cause of much controversy, since frequentist statisticians do not accept the parameters to be represented by random variables.

In Bayesian analysis, a prior probability distribution for all parameters in the statistical model is necessary. On observing that the prevalence of the disease is in a limited range $(0,1)$, a plausible prior probability distribution is as signed to a parameter $\theta$ given by a beta distribution 42 , a flexible probability distribution which can take many forms depending on the values of $a$ and $b$. In this case, the prior distribution $f(\theta)$ for $\theta$ has a probability density function given by $f(\theta)=\frac{1}{B(a, b)} \theta^{a-1}(1-\theta)^{b-1}, \quad 0<\theta<1$, where $a$ and $b$ are known values, and $B(a, b)$ is the beta distribution in which $a$ and $b$ are the hyperparameters of the prior distribution. Hyperparameters may be chosen by a panel of experts or by using results from previous studies. For example, let's say that an experienced epidemiologist believes that the prevalence of the disease in a specified population is around $15 \%$ and is almost certain that prevalence is no less than $2 \%$ and no greater than $30 \%$. Based on this information, we now need to find the values of the constants $a$ and $b$. One possible strategy is to approximate the mode of the beta distribution to $15 \%$ and 
its standard deviation to one quarter of the distance between the limits $2 \%$ and $30 \%$ (for further details see Browne 200143) thus giving $a=4.96$ and $b=23.45$. The graph in Figure 2 shows the probability density function of the beta distribution with parameter values of 4.96 and 23.45 mathematically representing the prior information given by the epidemiologist. It can be observed that the maximum value of the probability density function is $15 \%$ (the mode). In addition, it can be seen that the probability of prevalence being higher than $30 \%$ or lower than $2 \%$ is low. This process is called prior probability elicitation 44 .

The likelihood function plays a key role in statistical inference in both frequentist and Bayesian approaches. The Bayes' theorem says that the distribution of $\theta$ given the data (named posterior distribution) is proportional to the product of the prior distribution $f(\theta)$ and likelihood function $f(x \mid \theta)$. Bayes' formula establishes that $f(\theta \mid x) \propto f(\theta) \times f(x \mid \theta)$.

Thus, we have

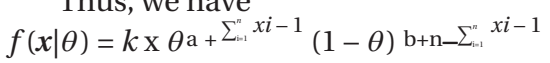

where $k$ is a constant value known as the normalizing constant. The posterior distribution for $\theta$ also follows a beta distribution, since the expression for $f(\theta \mid x)$ given above is in the form of a beta distribution. When the posterior distribution $f(\theta \mid x)$ is in the same family as the prior distribution $f(\theta)$, we say that $f(\theta)$ is a conjugate prior distribution for $\theta$
Let us suppose a sample of size $\mathrm{n}=100$ individuals from the population of interest, of which 22 individuals have the disease in interest. The maximum likelihood estimate for $\theta$ is given by $22 / 100=$ $22 \%$. Considering the Bayesian approach, the posterior distribution for $\theta$ is proportional to $f(\theta \mid x) \propto \theta^{4.96+22-1}(1-\theta)^{23.45+100-22-1}=\theta^{26.96-1}(1-\theta)^{101.45-1}$, since $a=4.96, b=23.45, \sum_{i=1}^{n} x_{i=22}$ and $n=100$. Thus, $f(\theta \mid x)$ follows a beta distribution with parameters 26.96 and 101.45 .

Figure 3 compares the prior and posterior distributions for $\theta$. We note that the curve that represents the posterior distribution is the lower dispersion curve, suggesting that the posterior distribution provides more information about $\theta$ than the prior distribution. Considering that the mean of a random variable that follows a beta distribution with parameters $a$ and $b$ is given by $a /(a+b)$, the Bayesian estimate of disease prevalence is given by $26.96 /(26.96+101.45)=21 \%$.

The graph in Figure 4 shows the posterior distribution for $\theta$, where the gray area corresponds to $95 \%$ of the total area under the curve. This area represents the $95 \%$ credible interval, which in this case is within a range of 14.4 to $28.4 \%$. The credible interval is the Bayesian equivalent of the frequentist confidence interval, and we can interpret that there is a $95 \%$ probability that the true prevalence $\theta$ lies within this range.

\section{Figure 2}

Prior probability distribution for $\theta$, given by a beta distribution with parameters 4.96 and 23.45 . The dashed line represents the mode of the distribution (maximum value).

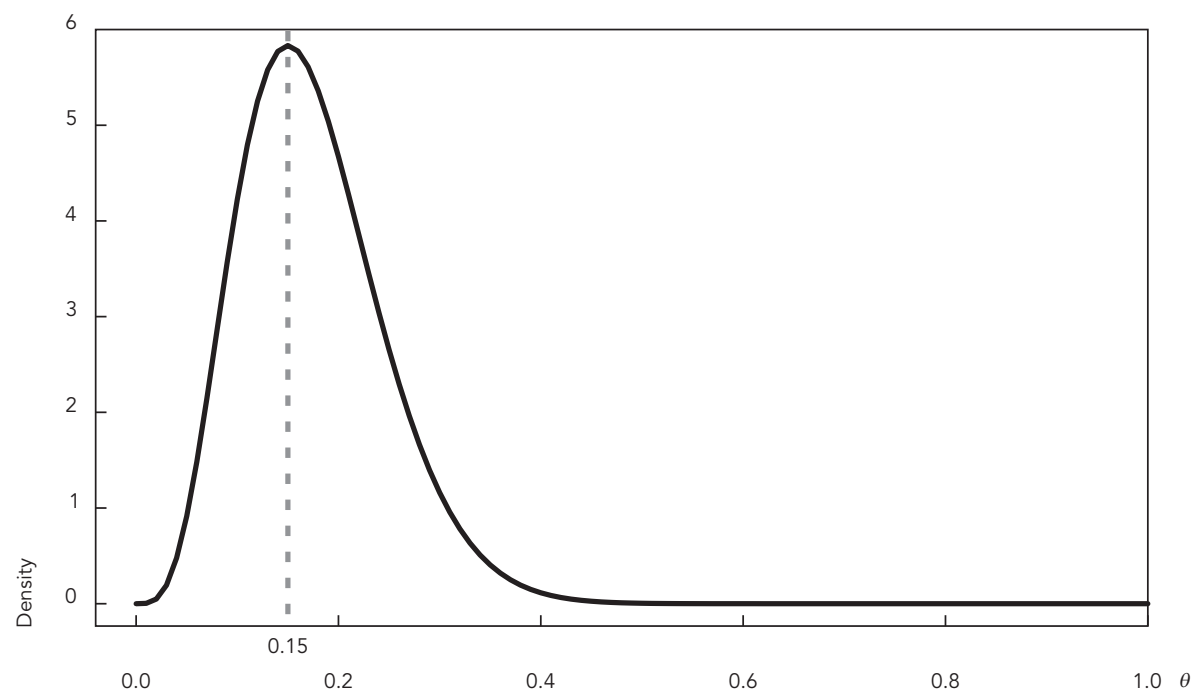


Figure 3

Comparison of prior probability distribution for $\theta$, given by a beta distribution with parameters 4.96 and 23.45 (dashed line) with the posterior distribution for $\theta$, given by a beta distribution with parameters 26.96 and 101.45 (continuous line).

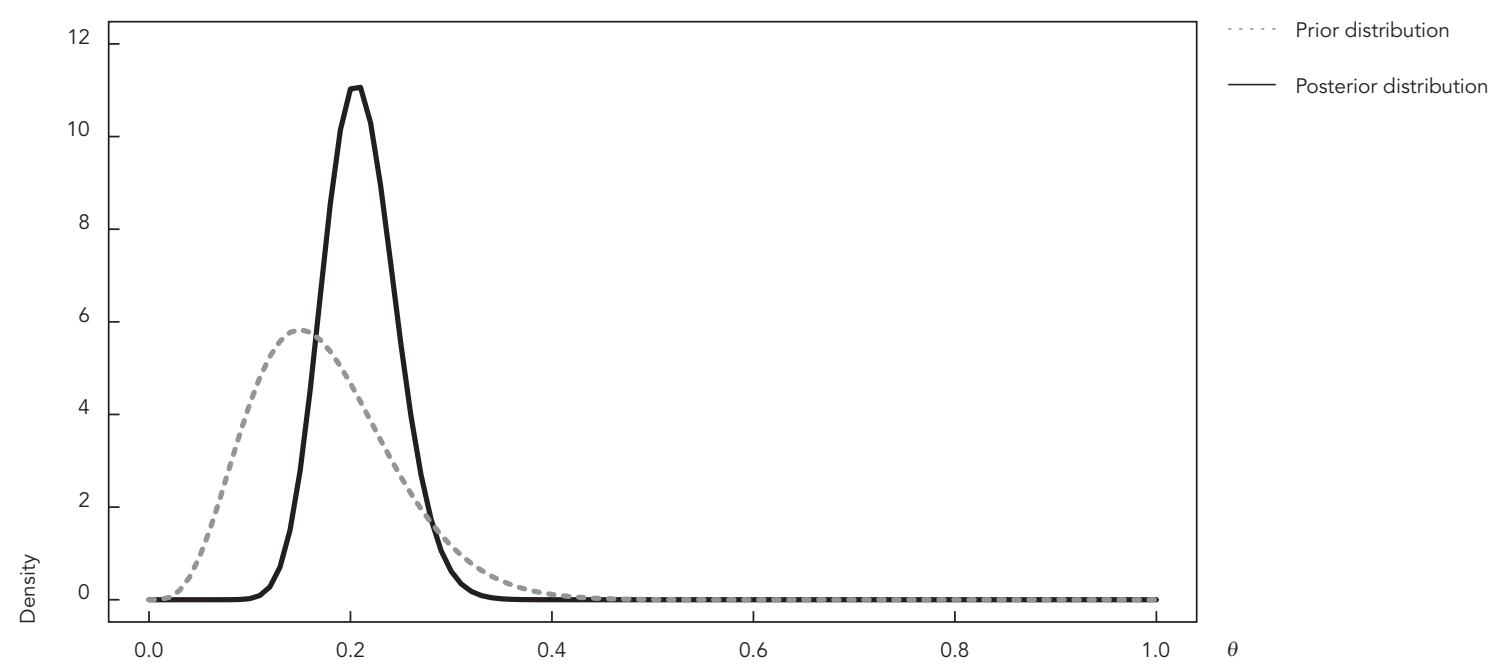

Figure 4

Posterior distribution for $\theta$ given by a beta distribution with parameters 26.96 and 101.45 , where the gray area describes a $95 \%$ credible interval.

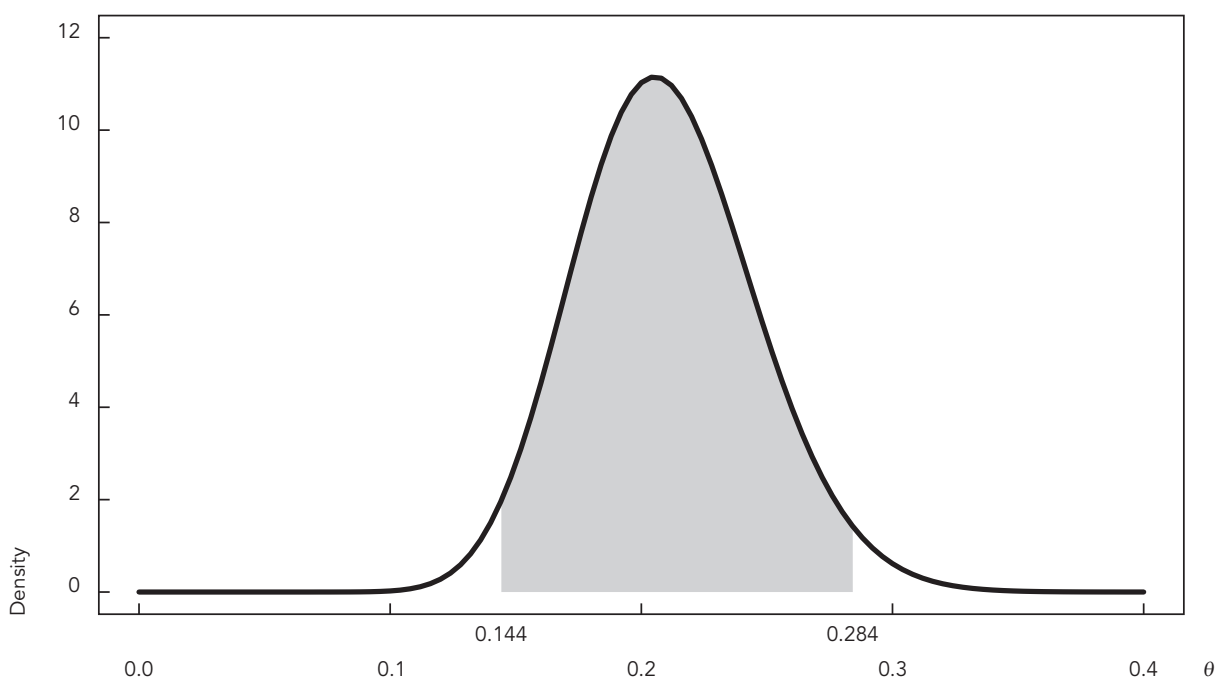




\section{The use of noninformative prior distribution}

The use of a noninformative prior distribution is suggested when there is total ignorance about the parameter of interest. When using noninformative prior distribution for the parameter $\theta$ the Bayesian estimates tend to be close to the corresponding maximum-likelihood estimates, since in this case $f(\theta)$ has minimal impact on the posterior distribution $f(\theta \mid x)$. There are different techniques for constructing noninformative prior distributions, such as the Jeffreys prior 45 based on the so-called Fisher information, a concept used in the theory of maximum-likelihood estimation. Using the problem presented in the previous section, the Jeffrey prior is defined in terms of a beta distribution with parameters 0.5 and 0.5 (see, for example, Box \& Tiao ${ }^{46}$ ). In this case, the posterior distribution $f(\theta \mid x)$ follows a beta distribution with parameters 22.5 and 78.5, and the Bayes estimate of prevalence is 22.5/ $(22.5+78.5)=22.3 \%$. Noninformative prior distributions are useful when we have no knowledge about the parameter of interest or when a more objective analysis is required. Bayesian analysis using noninformative prior distribution has a number of advantages over maximum-likelihood estimation in situations where the likelihood function is particularly complex and traditional optimization methods are not well suited to such problems.

Table 1 illustrates frequentist and Bayesian estimates (posterior means) of prevalence based on different sample sizes and choices of prior distribution for $\theta$. For all assumed sample sizes we fixed $\sum_{i=1}^{n} x_{i} / n=22 \%$. We also assigned beta (4.96, 23.45) as an informative prior distribution for $\theta$, beta $(0.5,0.5)$ as a noninformative prior distribution and beta $(10,10)$ as an example of an inadequate prior distribution based on an implausible expert opinion. Bayes estimates based on the noninformative prior distribution are similar to the frequentist estimates. As sample size increases, we can observe that frequentist and Bayes estimates become more similar, even in the case of the inadequate prior distribution for $\theta$. This occurs because in large samples the contribution of the likelihood function to the posterior distribution is relatively greater in relation to the adopted prior distribution for the parameter.

All calculations and simulations were carried out using the R software (The R Foundation for Statistical Computing, Vienna, Austria; http:// www.r-project.org).

Frequentist and Bayesian prevalence estimates (posterior means) based on different sample sizes and choices of prior distribution for $\theta$.

\begin{tabular}{|c|c|c|c|c|c|}
\hline \multirow[t]{4}{*}{$n$} & \multirow{4}{*}{${ }_{\mathrm{j}=1}^{n}$} & & \multicolumn{2}{|c|}{ Frequentist estimation } & Bayesian estimation \\
\hline & & & Prior $\theta \sim$ Beta $(4.96,23.45)$ & Prior $\theta \sim$ Beta $(0.5,0.5)$ & Prior $\theta \sim$ Beta $(10,10)$ \\
\hline & & Estimate & Estimate & Estimate & Estimate \\
\hline & & (95\% confidence interval) & (95\% credible interval) & (95\% credible interval) & (95\% credible interval) \\
\hline \multirow[t]{2}{*}{100} & 22 & 0.22 & 0.210 & 0.223 & 0.267 \\
\hline & & $(0.1388,0.3012)$ & $(0.1442,0.2842)$ & $(0.1475,0.3084)$ & $(0.1917,0.3489)$ \\
\hline \multirow[t]{2}{*}{150} & 33 & 0.22 & 0.213 & 0.222 & 0.253 \\
\hline & & $(0.1537,0.2863)$ & $(0.1560,0.2756)$ & $(0.1595,0.2912)$ & $(0.1906,0.3207)$ \\
\hline \multirow[t]{2}{*}{200} & 44 & 0.22 & 0.214 & 0.221 & 0.245 \\
\hline & & $(0.1626,0.2774)$ & $(0.1637,0.2697)$ & $(0.1668,0.2812)$ & $(0.1909,0.3043)$ \\
\hline \multirow[t]{2}{*}{250} & 55 & 0.22 & 0.215 & 0.221 & 0.241 \\
\hline & & $(0.1687,0.2713)$ & $(0.1691,0.2654)$ & $(0.1720,0.2744)$ & $(0.1917,0.2934)$ \\
\hline \multirow[t]{2}{*}{500} & 110 & 0.22 & 0.218 & 0.221 & 0.231 \\
\hline & & $(0.1837,0.2563)$ & $(0.1834,0.2537)$ & $(0.1853,0.2579)$ & $(0.1956,0.2679)$ \\
\hline \multirow[t]{2}{*}{1,000} & 220 & 0.22 & 0.219 & 0.220 & 0.225 \\
\hline & & $(0.1943,0.2457)$ & $(0.1940,0.2445)$ & $(0.1951,0.2465)$ & $(0.2003,0.2516)$ \\
\hline
\end{tabular}




\section{Advantages of Bayesian methods}

Several authors claim that the main advantage of the Bayesian approach over the frequentist method is that it allows the incorporation of prior knowledge by specifying appropriate prior probabilities 47,48. However, the advantages of Bayesian methods are not limited to the possibility of incorporating out-of-sample information into the analyses. For example, Bayesian methods are especially useful for statistical inference of complex models which present significant difficulties for frequentist methods. Calculating the maximum of very complex likelihood functions can be a difficult task in practice, despite the advances in computer software and hardware in recent years. In such situations, the frequentist approach usually involves numerical tools, such as the traditional Newton-Raphson method. However, convergence problems may occur or solutions may be highly dependent on initial values. Bayesian methods can overcome this problem by using the MCMC 49,50 methods, that allow samples to be simulated using the parameters of interest. In this approach, inference is therefore based on the sample, remembering that the Bayesian approach treats the parameters as random variables. In some special situations, this procedure can be simplified by the use of a Bayesian technique based on a procedure called data augmentation introduced by Tanner \& Wong 51. This procedure "augments" the observed data to simplify the likelihood function.

Another important aspect of the frequentist inference approach concerns the identifiability of the parameters of a given model. The problem of identifiability occurs when there are more parameters than degrees of freedom. In such situations, parameter estimation based on the frequentist approach is a difficult task. Degrees of freedom can be understood as "the number of independent units of information in a sample relevant to the estimation of a parameter or calculation of a statistic" 52 (p. 118). A practical example is the assessment of new diagnostic tests which have not achieved the gold standard. Joseph et al. 53 showed that in these types of situations, where there are more parameters (sensitivity, specificity and disease prevalence) than information from the data, Bayesian methods are able to provide estimates for these measures.

Although the literature includes studies that used Bayesian hypothesis testing, the main focus of the Bayesian method is estimating parameters and not hypothesis testing. For example, given two hypotheses $\mathrm{H}_{1}$ and $\mathrm{H}_{2}$, a Bayesian hypothesis test compares the probability of the observed data $\mathrm{D}$ given $\mathrm{H}_{1}$, denoted by $\mathrm{P}\left(\mathrm{D} \mid \mathrm{H}_{1}\right)$, and the prob- ability of the observed data $\mathrm{D}$ given $\mathrm{H}_{2}$, denoted by $\mathrm{P}\left(\mathrm{D} \mid \mathrm{H}_{2}\right)$. The ratio $\mathrm{BF}=\mathrm{P}\left(\mathrm{D} \mid \mathrm{H}_{1}\right) / \mathrm{P}\left(\mathrm{D} \mid \mathrm{H}_{2}\right)$ is the Bayes factor 54 , which quantifies the evidence from data for $\mathrm{H}_{1}$ in relation to $\mathrm{H}_{2}$. It should be noted that this procedure is different from the traditional null hypothesis significance testing. While the results of frequentist hypothesis tests are usually expressed as p-values, the results of Bayesian hypothesis tests are expressed as Bayes factors. P-values are difficult to interpret and are regularly misinterpreted by health researchers, while Bayes factors are more easy to interpret.

\section{Recent trends in Bayesian analysis}

The following is non-exhaustive list of areas of epidemiological research which should benefit from the use of Bayesian methods over the coming years.

\section{Spatiotemporal modeling}

Ecological studies involve the description of the geographical distribution of a disease or an event of interest and associated factors. In this context, spatial autoregressive models have been extensively used in data analysis and a popular modeling approach has been through the conditionally autoregressive (CAR) distribution and their generalizations. These models are relatively flexible and can accommodate different structures of spatial correlation and longitudinal data, as well as the presence of covariates. The estimation of the parameters of these models based on frequentist inference methods can be a difficult task due to the complexity of the likelihood function, and Bayesian methods provide a convenient alternative to deal with this model structure. This type of modeling is facilitated by the use of the software OpenBUGS, that allows sample simulation for CAR distribution and multivariate extension 55,56 . In a broader sense, these spatiotemporal models 57 can be classified as a type of hierarchical model. Multilevel or hierarchical models are useful for the analysis of data structured in groups, which is common in epidemiological studies.

\section{Models based on distributions rather than normal curve}

The use of models based on normal distribution is quite common in epidemiological studies. There is a belief that nonparametric hypothesis testing should be used when the normality assumption is not satisfied, but this is not necessarily true due to the existence of generalized linear 
models (GLM), a very general class of statistical models that includes many probability distributions. In addition, traditional nonparametric tests only provide $\mathrm{p}$-values, while measures of the size of the association between groups are essential for epidemiological research. The relationship between dependent discrete variables and explanatory variables can be explored by using models based on Poisson, binomial, negative binomial or beta-binomial distributions, depending on the amount of data dispersion. Count data with excess zeros 58 and truncated data 59 are also common in epidemiological studies and specific regression models are required to deal with this. Bayesian methods can be very useful in these modeling applications, since they enable us to estimate parameters and related measures of association in complex models or where asymptotic assumptions are not appropriate due to sparse data or small sample sizes.

\section{Models for survival data based on more complex distribution}

In epidemiological studies, parametric survival models are usually based on the Weibull, lognormal or gamma distributions. Alternative distributions for time-to-event data have been used by studies mentioned in the literature in recent years, allowing the addition of a parameter representing the proportion of individuals which are "immune" to the event of interest 60 . These distributions are extensions of usual distributions including a greater number of unknown parameters 61,62 . Parameter estimation in survival models based on these distributions can be challenging, especially when covariates are involved, since asymptotic properties cannot be assured. Bayesian analysis could be a promising alternative for this type of modeling because the use of MCMC methods are capable of dealing with the complexity of the resulting likelihood function.

\section{Multivariate copula models}

Copula functions 63 are tools used to construct and simulate multivariate distributions. For example, copula functions can be used to study the joint distribution of the successive survival times 64 , multiple dependent diagnostic tests 65 or the association of risk factors for two or more diseases simultaneously. Bayesian methods can accommodate different copula functions and may therefore be useful for many epidemiological investigations that use multivariate data.

\section{Concluding remarks}

Since Bayesian methods allows the incorporation of relevant prior knowledge or beliefs into the analysis, the researcher is no longer just an observer in the research process and his or her experience becomes an active component to obtain inferences of interest. This in itself is often seen as a controversial aspect of Bayesianism, since the traditional scientific method relies on a positivist approach and has been proposed to avoid subjective analysis. The Bayesian method offers a different way of thinking about research and we believe that it can make a valuable contribution to the development of knowledge in a number of fields apart from epidemiology.

From a statistical viewpoint, we believe that the major advantage of the Bayesian approach is its extreme flexibility. The availability of MCMC methods allows the analysis of a wide range of statistical models which could be applied to epidemiologic research, such as hierarchical models, longitudinal models and more complex models applied to specific design studies 66,67 or unusual data structures.

Currently, good Bayesian analysis software is available, such as OpenBugs, SAS and several $\mathrm{R}$ software libraries. An important advantage of OpenBugs and R programs is that they are freely available on the internet. However, the use of these programs requires some knowledge of programming language. Therefore, researchers who are not proficient in computer programming may have some difficulties with Bayesian modeling, and this is an obstacle to popularizing Bayesian methods in epidemiological research. This situation may also be aggravated by a lack of professional statistical support in health research institutions.

Despite these potential difficulties, this study observed a sharp increase in the number of studies using Bayesian methods and this trend looks set to continue. It can therefore be concluded that epidemiologists, clinicians and health professions students interested in a research career should receive appropriate training in Bayesian methods to be able to deal with more complex problems. 


\section{Resumen}

Durante el año 2013 se conmemora el 250 aniversario de la presentación del teorema de Bayes por el filósofo Richard Price ante la Royal Society en 1763. Thomas Bayes era una persona poco conocida en su época, pero en el siglo XX el teorema que lleva su nombre se utilizó ampliamente en muchos campos de investigación. El teorema de Bayes es la base de los llamados métodos bayesianos, procedimiento de inferencia estadística que permite incorporar en el análisis el conocimiento previo acerca de las características relevantes de los datos. En la actualidad, los métodos bayesianos son ampliamente utilizados en muchas áreas diferentes, tales como la astronomía, la genética, la bioinformática y las ciencias sociales. Muchos autores han discutido los recientes avances en el uso de métodos bayesianos en el análisis de los datos epidemiológicos. En este artículo se presenta una visión general de los métodos bayesianos, su utilidad en la investigación y en epidemiología en donde los métodos bayesianos pueden utilizarse extensamente durante los próximos años.

Teorema de Bayes; Estadística; Teoría de la Probabilidad

\section{Contributors}

E. Z. Martinez and J. A. Achcar contributed to project design, the literature review and writing and revision of this article.

\section{Acknowledgments}

The authors are very grateful to the editor and referees for their constructive criticism and suggestions, which helped improve this paper. Both authors were supported by fellowships from the CNPq.

\section{References}

1. Bayes T. An essay towards solving a problem in the doctrine of chances. Philos Trans R Soc Lond 1763; 53:370-418.

2. Holland JD. The Reverend Thomas Bayes, F.R.S. (1702-61). J R Stat Soc Series A 1962; 125:451-61.

3. Bellhouse DR. The Reverend Thomas Bayes, FRS: a biography to celebrate the tercentenary of his birth. Stat Sci 2004;19:3-43.

4. Pomeroy RS. Hume on the testimony for miracles. Speech Monographs 1962; 29:1-12.

5. Holder RD. Hume on miracles: Bayesian interpretation, multiple testimony, and the existence of God. Br J Philos Sci 1998; 49:49-65.

6. Owen D. Hume versus Price on miracles and prior probabilities: testimony and the Bayesian calculation. Phil Q 1987; 37:187-202.

7. Sobel JH. On the evidence of testimony for miracles: a Bayesian interpretation of David Hume's analysis. Phil Q 1987; 37:166-86.

8. Androutsopoulos I, Koutsias J, Chandrinos KV, Paliouras G, Spyropoulos CD. An evaluation of naive Bayesian anti-spam filtering. In: Proceedings of the Workshop on Machine Learning in the New Information Age. 11th European Conference on Machine Learning. New York: Springer-Verlag; 2000. p. 9-17.

9. Pedersen L. Autonomous characterization of unknown environments. IEEE Int Conf Robot Autom 2001; 1:277-84.

10. Pedersen L, Wagner M, Apostolopoulos D, Whittaker WR. Autonomous robotic meteorite identification in Antarctica. IEEE Int Conf Robot Autom 2001; 1:4158-65.

11. Halatci I, Brooks CA, Iagnemma K. Terrain classification and classifier fusion for planetary exploration rovers. In: Proceedings of the Aerospace Conference, 2007 IEEE. Big Sky: Institute of Electrical and Electronics Engineers; 2007. p. 1-11. 
12. Jenkins CR, Peacock JA. The power of Bayesian evidence in astronomy. Mon Not R Astron Soc 2011; 413:2895-905.

13. Koop G, Poirier DJ, Tobias JL. Bayesian econometric methods. Cambridge: Cambridge University Press; 2007.

14. Lancaster T. Introduction to modern Bayesian econometrics. Oxford: Wiley-Blackwell; 2004.

15. Rossi PE, Allenby GM. Bayesian statistics and marketing. Marketing Science 2003; 22:304-28.

16. Makov UE. Principal applications of Bayesian methods in actuarial science: a perspective. $\mathrm{N}$ Am Actuar J 2001; 5:53-7.

17. Edwards W, Lindman H, Savage LJ. Bayesian statistical inference for psychological research. Psychol Rev 1963; 70:193-242.

18. Beaumont MA, Rannala B. The Bayesian revolution in genetics. Nat Rev Genet 2004; 5:251-61.

19. Shoemaker JS, Painter IS, Weir BS. Bayesian statistics in genetics: a guide for the uninitiated. Trends Genet 1999; 15:354-8.

20. Huelsenbeck JP, Ronquist F, Nielsen R, Bollback JP. Bayesian inference of phylogeny and its impact on evolutionary biology. Science 2001; 294:2310-4.

21. Wilkinson DJ. Bayesian methods in bioinformatics and computational systems biology. Brief Bioinform 2007; 8:109-16.

22. Daponte BO, Kadane JB, Wolfson LJ. Bayesian demography: projecting the Iraqi Kurdish population, 1977-1990. J Am Stat Assoc 1997; 92:1256-67.

23. Jackman S. Bayesian analysis for the social sciences. New York: John Wiley \& Sons; 2009.

24. Etzioni RD, Kadane JB. Bayesian statistical methods in public health and medicine. Annu Rev Public Health 1995; 16:23-41.

25. Gupta SK. Use of Bayesian statistics in drug development: advantages and challenges. Int J Appl Basic Med Res 2012; 2:3-6.

26. Lewis RJ, Wears RL. An introduction to the Bayesian analysis of clinical trials. Ann Emerg Med 1993; 22:1328-36.

27. Zhang X, Cutter G. Bayesian interim analysis in clinical trials. Contemp Clin Trials 2008; 29:751-5.

28. Dunson DB. Commentary: practical advantages of Bayesian analysis of epidemiologic data. Am J Epidemiol 2001; 153:1222-6.

29. Greenland S. Bayesian perspectives for epidemiological research: I. Foundations and basic methods. Int J Epidemiol 2006; 35:765-75

30. Greenland S. Bayesian perspectives for epidemiological research. II. Regression analysis. Int J Epidemiol 2007; 36:195-202.

31. Greenland S. Bayesian perspectives for epidemiologic research: III. Bias analysis via missing-data methods. Int J Epidemiol 2009; 38:1662-73.

32. Congdon P. Applied Bayesian modelling. New York: John Wiley \& Sons; 2003.

33. Moore DS. Bayes for beginners? Some reasons to hesitate. Am Stat 1997; 51:254-61.

34. Shuford Jr. EH. Some Bayesian learning processes. Tech Doc Rep U S Air Force Syst Command Electron Syst Div 1963; 86:1-39.

35. Lunn DJ, Thomas A, Best N, Spiegelhalter D. WinBUGS: a Bayesian modelling framework: concepts, structure, and extensibility. Stat Comput 2000; 10:325-37.
36. Gelfand AE, Smith AF. Sampling-based approaches to calculating marginal densities. J Am Statist Assoc 1990; 85:398-409.

37. Lykou A, Ntzoufras I. WinBUGS: a tutorial. WIREs Computational Statistics 2001; 3:385-96.

38. Adamina M, Tomlinson G, Guller U. Bayesian statistics in oncology: a guide for the clinical investigator. Cancer 2009; 115:5371-81.

39. Basáñez MG, Marshall C, Carabin H, Gyorkos T, Joseph L. Bayesian statistics for parasitologists. Trends Parasitol 2004; 20:85-91.

40. Casella G, Berger RL. Statistical inference. 2nd Ed Farmington Hills: Cengage Learning; 2001.

41. Cox DR. Principles of statistical inference. Cambridge: Cambridge University Press, 2006.

42. Krishnamoorthy K. Handbook of statistical distributions with applications. Boca Raton: Chapman \& Hall; 2006.

43. Browne RH. Using the sample range as a basis for calculating sample size in power calculations. Am Stat 2001; 55:293-8.

44. O'Hagan A, Buck CE, Daneshkhah A, Eiser JR, Garthwaite PH, Jenkinson DJ, et al. Uncertain judgements: eliciting experts' probabilities. Chichester: John Wiley \& Sons; 2006.

45. Jeffreys $\mathrm{H}$. An invariant form for the prior probability in estimation problems. Proc R Soc A 1946; 186:453-61.

46. Box GEP, Tiao GC. Bayesian inference in statistical analysis. New York: John Wiley \& Sons; 1992.

47. Gunel E, Wearden S. Bayesian estimation and testing of gene frequencies. Theor Appl Genet 1995; 91:534-43.

48. Matawie KM, Assaf A. Bayesian and DEA efficiency modelling: an application to hospital foodservice operations. J Appl Statist Sci 2010; 37:945-53.

49. Smith AFM, Roberts GO. Bayesian computation via the Gibbs sampler and related Markov chain Monte Carlo methods. J R Stat Soc Series B 1993; 55:3-23.

50. Brooks S. Markov chain Monte Carlo method and its application. J R Stat Soc Series D 1998; 47: 69-100.

51. Tanner MA, Wong WH. The calculation of posterior distributions by data augmentation. J Am Statist Assoc 1987; 82:528-40.

52. Everitt BS. The Cambridge dictionary of statistics. 3rd Ed. Cambridge: Cambridge University Press; 2006.

53. Joseph L, Gyorkos TW, Coupal L. Bayesian estimation of disease prevalence and the parameters of diagnostic tests in the absence of a gold standard. Am J Epidemiol 1995; 141:263-72.

54. Masson MEJ. A tutorial on a practical Bayesian alternative to null-hypothesis significance testing. Behav Res Methods 2011; 43:679-90.

55. Branscum AJ, Perez AM, Johnson WO, Thurmond MC. Bayesian spatiotemporal analysis of foot-andmouth disease data from the Republic of Turkey. Epidemiol Infect 2008; 136:833-42.

56. Roza DL, Caccia-Bava MC, Martinez EZ. Spatiotemporal patterns of tuberculosis incidence in $\mathrm{Ri}$ beirão Preto, State of São Paulo, southeast Brazil, and their relationship with social vulnerability: a Bayesian analysis. Rev Soc Bras Med Trop 2012; 45:607-15 
57. Banerjee S, Gelfand AE, Carlin BP. Hierarchical modeling and analysis for spatial data. Boca Raton: CRC Press; 2003.

58. Lewsey JD, Thomson WM. The utility of the zeroinflated Poisson and zero-inflated negative binomial models: a case study of cross-sectional and longitudinal DMF data examining the effect of socio-economic status. Community Dent Oral Epidemiol 2004; 32:183-9.

59. Brookmeyer R, Blades N, Hugh-Jones M, Henderson DA. The statistical analysis of truncated data: application to the Sverdlovsk anthrax outbreak. Biostatistics 2001; 2:233-47.

60. Chen MH, Ibrahim JG, Sinha D. Bayesian inference for multivariate survival data with a cure fraction. J Multivar Anal 2002; 80:101-26.

61. Carrasco JMF, Ortega EMM, Cordeiro GM. A generalized modified Weibull distribution for lifetime modeling. Comput Stat Data Anal 2008; 53:450-62.

62. Barreto-Souza W, Morais AL. The Weibull-geometric distribution. J Stat Comput Simul 2011; 81: 645-57.
63. Nelsen RB. An introduction to copulas. New York: Springer-Verlag; 1999.

64. Romeo JS, Tanaka NI, Pedroso-de-Lima AC. Bivariate survival modeling: a Bayesian approach based on copulas. Lifetime Data Anal 2006; 12:205-22.

65. Tovar JR, Achcar JA. Dependence between two diagnostic tests with copula function approach: a simulation study. Commun Stat Simul Comput 2013; 42:454-75.

66. Zelen M, Parker RA. Case-control studies and Bayesian inference. Stat Med 1986; 5:261-9.

67. Ghosh M, Song J, Forster JJ, Mitra R, Mukherjee B. On the equivalence of posterior inference based on retrospective and prospective likelihoods: application to a case-control study of colorectal cancer. Stat Med 2012; 31:2196-208.

Submitted on 08/Aug/2013

Final version resubmitted on 22/Nov/2013

Approved on 26/Nov/2013 
Martinez EZ, Achcar JA. Trends in epidemiology in the 21st century: time to adopt Bayesian methods. Cad Saúde Pública 2014; 30(4): 703-714.

A revista foi informada um erro na equação que descreve o teorema de Bayes (p. 707). A equação correta é:

The journal has been informed of an error in the equation that describes the Bayes' theorem ( $\mathrm{p}$. 707). The correct equation is:

La revista fue informada sobre un error en la ecuación que describe el teorema de Bayes (p. 707). La ecuación correcta es:

$f(\theta \mid x) \propto f(\theta) \times f(x \mid \theta)$.

A revista foi informada um erro no oitavo parágrafo da seção A Practical Example: Estimating Disease Prevalence (p. 707). O parágrafo correto é:

The journal has been informed of an error in the eighth paragraph of the section A Practical Example: Estimating Disease Prevalence (p. 707). The correct paragraph is:

La revista fue informada sobre un error en el octavo párrafo de la sección A Practical Example: Estimating Disease Prevalence (p. 707). El párrafo correcto es:

Let us suppose a sample of size $\mathrm{n}=100$ individuals from the population of interest, of which 22 individuals have the disease in interest. The maximum likelihood estimate for $\theta$ is given by $22 / 100=$ $22 \%$. Considering the Bayesian approach, the posterior distribution for $\theta$ is proportional to $f(\theta \mid x) \propto \theta^{4.96+22-1}(1-\theta)^{23.45+100-22-1}=\theta^{26.96-1}(1-\theta)^{101.45-1}$, since $a=4.96, b=23.45, \sum_{i=1}^{n} x_{i=22}$ and $n=100$. Thus, $f(\Theta \mid x)$ follows a beta distribution with parameters 26.96 and 101.45. 